\title{
A Neuro-inspired Adaptive Motion Detector
}

\author{
Xiaopin Zhong ${ }^{1}$, Lin $\mathrm{Ma}^{2}$ \\ ${ }^{1}$ College of Mechatronics and Control Engineering, Shenzhen University, Shenzhen, China \\ ${ }^{2}$ Shaanxi international trust co.,ltd. Xian’an, China \\ Email: xzhong@szu.edu.cn, linn.mars@gmail.com
}

Received 2013

\begin{abstract}
In this paper, a novel motion detector is proposed to perceive the weak changes in a image sequence. This is inspired by the mechanism of fixational eye movement and dynamics of vertebrate's cortex. We realized respectively an artificial model of visual attention selection, called dual-probe adaptive model (DPAM), and an active tremor operation (ATO) approach. It is found that between them there exists a resonance phenomenon. The phenomenon is enhanced when the ATO and the DPAM are in-phase and is suppressed when they are anti-phase. Based on this, we construct a novel motion detector combined by the ATO and the DPAM to resonate with the motion direction. This allows capturing moving edges even in the image sequences with lighting change and noisy background. Simulation and Experimental results demonstrate the effectiveness.
\end{abstract}

Keywords: Neuro-inspired; Motion Detector; Dual-probe Adaptive Model; Active Tremor Operation

\section{Introduction}

Motion detection is an important basic process in many video analysis tasks [1], such as object detection, behavior recognition and video encoding. There exist a number of typical approaches for motion detection. However, they consider in an image sequence all of the areas instead of the areas with motion.

The most used two well-known methods are the temporal difference [2] and the background modeling [3]. They can work well only when the background is approximately stationary and the foreground is relatively moving, i.e. they are sensitive to noise and variations in illumination.

Marr-Ullman model [4] is an early motion detector which realized a highly sensitive 1D directional-selective detection by using the temporal derivative of zero-crossing fragment of measurement. This detector passively responds to any significantly-moving object.

The optic flow [5] is also a successful visual motion detection technique. There have recently emerged some specific computing techniques for highly accurate optic flows, but then they are still computationally inefficient.

In fact, human and primate's visual system can not only localize accurately moving objects, perceive their moving direction and velocity. This offers the cognitive ability to make use of the limited computing resource. Bouzerdoum and Pinter [6,7] proposed a directional selective multiplicative inhibitory motion detector (MIMD) under steady lighting condition. Based on MIMD, Wang and Zheng [8] further developed a multiplicative inhibi- tory velocity detector (MIVD) by replacing the low-pass filter with a band-pass filter to determine the detectors temporal feature. This replacement allows obtaining the selectivity of motion vector for a motion detector.

Inspiring by the Sterlings model of the retinal nerve circuits [9], a dynamic differential equation groups has been set up by Ma et al. [10], which could be used to capture the weak changing signals exactly in noisy background with few parallel computing steps.

In the authors' previous research [11], a group of dynamic differential equations has been set up to capture exactly the weak changing signals in noisy backgrounds. It is so-called the Dual-Probe Adaptive Model (DPAM).

Inspired by the mechanism of fixational eye movements of human vision [13,14], we simulated an active tremor operation (ATO). It is found that there is a resonance phenomenon between the ATO and the DPAM. This allows perceiving the motion direction. Based on this finding, we propose then an adaptive motion detector. This detector can perceive edges with specific moving directions and adapt to changes of background and lighting.

The paper is structured as follows. In Section II, the detailed adaptive motion detector is introduced and discussed. In Section III simulation and experimental results are shown and analyzed and we conclude in Section IV.

\section{Adaptive Motion Detector}

\subsection{Dual-Probe Adaptive Model (DPAM)}

In fact, the so-call DPAM is a family of dynamic spatial 
temporal filters, and the parameter settings determine the actual performance of a corresponding detector in response to the input video. We denote first the model by the equations as follows.

$$
\begin{gathered}
\left\{\begin{array}{l}
s_{i}(k)=a_{s s} \cdot s_{i}(k-1)+a_{s h} \cdot h_{i}(k-1)+b_{s} \cdot \tilde{u}_{i}(k) \\
h_{i}(k)=a_{h s} \cdot\left\langle\mathbf{s}(k), \mathbf{K}_{h, i}\right\rangle+a_{h h} \cdot h_{i}(k-1)
\end{array}\right. \\
A_{i}(k)=\left\langle\mathbf{A}(k-1), \mathbf{K}_{A, i}\right\rangle_{i}-\alpha_{A} \cdot\left(A_{i}(k-1)-h_{i}(k)\right) \\
\qquad \begin{array}{l}
b_{i}^{H}(k)=s_{i}(k)-h_{i}(k) \\
b_{i}^{D}(k)=-b_{i}^{H}(k) \\
\bar{b}_{i}^{H}(k)=\left(\mathbf{b}^{H}(k) * \mathbf{K}_{b}\right)_{i} \\
\bar{b}_{i}^{D}(k)=\left(\mathbf{b}^{D}(k) * \mathbf{K}_{b}\right)_{i}
\end{array} \\
\left\{\begin{array}{l}
G_{i}^{+}(k)=b_{i}^{D}(k)-\left(A_{i}(k)+\bar{b}_{i}^{H}(k)\right) \\
G_{i}^{-}(k)=b_{i}^{H}(k)-\left(A_{i}(k)+\bar{b}_{i}^{D}(k)\right)
\end{array}\right.
\end{gathered}
$$

where $u_{i}(k), \quad i=1, \cdots, N$ denotes the $i^{\text {th }}$ component of the input intensity vector $\mathbf{u}(k) . s_{i}(k)$ and $h_{i}(k)$ stand for the $i^{t h}$ component of the state variable feedforward vector $\mathbf{s}(k)$ and feedback vector $\mathbf{h}(k)$ respectively. $\quad \mathbf{K}_{h, i}>0$ is the unit vector of coefficient of the lateral inhibition effect of $h_{i}$ to $s_{j}$ in the surrounding of $G_{i}(k)$ pathway. This is to simulate a local grade potential of nerve cells. $b_{s}>0$ denotes the gain coefficient of photoreceptor. $a_{h s}$ and $a_{s h}$ represent the transfer coefficient of $s_{i}(k)$ and the feedback coefficient of $h_{i}(k-1)$ to $s_{i}(k) . a_{h s}>0$ and $a_{h h} \geq 0$ are the coefficient of renewal equation and confined to the convex combination $a_{h h}+a_{h s}=1$.

The formula 2 is an adaptive threshold equation, where $A_{i}(k)$ is the $i^{\text {th }}$ component of vector of $\mathbf{A}(k)$, denotes the adaptive threshold; $\alpha_{A} \neq 0$ is the coefficient of renewal equation; and $\mathbf{K}_{A, i}\left(\mathbf{K}_{A, i}>0\right)$ is the unit vectors of coefficient of the lateral inhibition effect of $A_{i}(k)$ to $A_{j}(k)$ in the surrounding of $G_{i}(k)$ pathway, which is similar to $\mathbf{K}_{h, i}$ and wider than it.

For convenience, we define in formula 3 four intermediate variables $b_{i}^{H}(k), b_{i}^{D}(k), \bar{b}_{i}^{H}(k)$ and $\bar{b}_{i}^{D}(k)$ to build respectively the corresponding vectors $\mathbf{b}^{H}(k)$, $\mathbf{b}^{D}(k), \mathbf{b}^{H}(k)$ and $\mathbf{b}^{D}(k)$. They are also known as bipolar variables named after bipolar cell of retina.

The formula 4 is exactly the dual push-pull probes output equation of the model, where $\mathbf{G}^{+}(k)$ and $\mathbf{G}^{-}(k)$ are the output dual probes of the model. The readers are referred to [11] for the analysis and discussion of the model parameters.

\subsection{Active Tremor Operation Based Detector}

In the neurobiological research, three types of unconscious eye movements are regarded during gazing a target. They are high frequency tremor, microsaccades and slow drifts $[12,16]$. Further research illustrates that the role of microsaccades and drifts is to latch down objects and to compensate the noisy control of muscle [12]. Only the tremor is believed to be related to visual perception, i.e. visual fading on retina is inhibited by tremors [15].

Inspired by this point, we introduce an active tremor operation (ATO) into the DPAM model [10,11]. According to the analysis in reference [11], when the update coefficient is close to zero, the DPAM is reduced to a typical image change detector. It is called DPAM-m detector because the output is like an $\mathrm{M}$ type cell of human retina [18]. With this additional ATO, the selective-inhibitory visual fading is then realized in a DPAM-m detector. We call this the ATO-DPAM-m detector in the followings. See Figure 1 for the processing structure.

For convenience, we redefine the ATO and introduce the basic process.

Definition 1: Active Tremor Operation (ATO) is a periodic translational operation of global image on digital video flows. This operation repeats a same translation of all pixels of a frame with an identical direction, amplitude and frequency.

As shown in Figure 2, for video flow $u(k)$ constructed by image sequence $I(k)$, suppose that the translation amplitude and the frequency of ATO are $\Delta d(k)$ and 0.25 round/s respectively, i.e.

$$
\tilde{u}_{p}(k)=u_{p+\Delta d(k)}(k),
$$

where $p$ denotes the coordinates of a pixel in image $I$, and $\Delta d(k)$ is a function of time $k$.

In the case of ATO-DPAM-m detector, the push-pull outputs denoted by $\tilde{\mathbf{G}}^{+}(k)$ and $\tilde{\mathbf{G}}^{-}(k)$ mean the Ontype response and the Off-type response respectively.

We found that there exists a resonance in the response of an ATO-DPAM-m detector. This is because in the DPAM-m detector, a visual fading occurs for the part without change in the image sequence and an enhancement response occurs for the part with change. In other words, ATO enhances DPAM if they are in-phase and

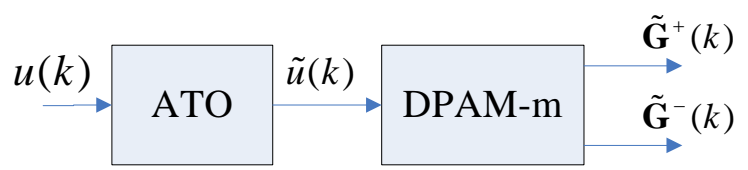

Figure 1. Structure of ATO-DPAM-m detector.

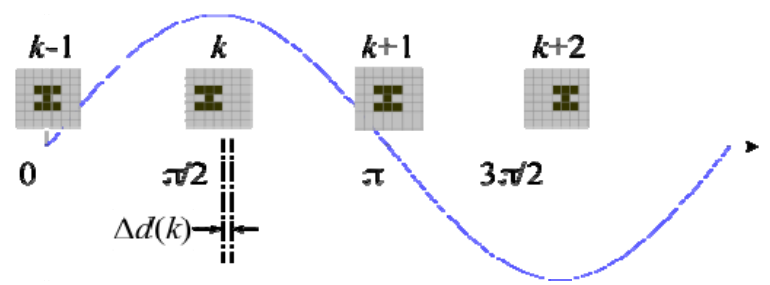

Figure 2. An example of ATO process. 
ATO inhibits DPAM if they are anti-phase. Therefore, the output of ATO-DPAM-m detectors has the selective ability and is highly related to the direction and the phase of ATO. This provides a solid foundation of motion detectors with directional selectivity.

To differentiate the directions, we use subscript •, $(\bullet=P, N)$ for the responds, i.e. $\tilde{\mathbf{G}}_{\bullet}^{+}(k)$ and $\tilde{\mathbf{G}}_{\bullet}^{-}(k)$, where $\mathrm{P}$ means the positive operational direction and $\mathrm{N}$ for the negative one. As shown in Figure $\mathbf{3}$ for example, it is shown in a synthesized image sequence $u_{p}(k)=I_{p+k v}^{\prime}$ that there occurs in-phase enhancement or anti-phase inhibition according to the relationship between $\Delta d$ and velocity $v$, and the ATO phases.

\subsection{Adaptive Motion Detector (AMD)}

We consider further a pair of ATO-DPAM-m detectors with inverse directions, i.e. a positive directional detector called ATOP-DPAM- $m$ and a negative one called ATON-DPAM-m. See in Figure 4 for the configuration. The parameters of both detectors are all identical except that the difference of their phase is $\pi$ and particularly $a_{h s}$ is set to 0.01 . We then obtain a new ATO-based adaptive motion detector.

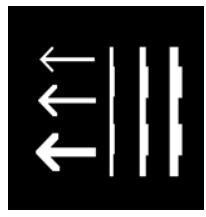

(a)

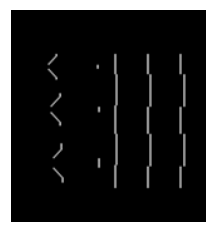

(e)

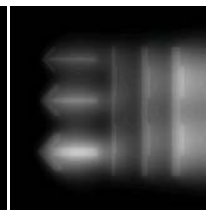

(b)

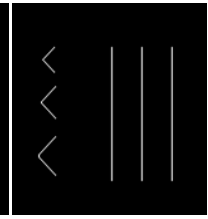

(f)

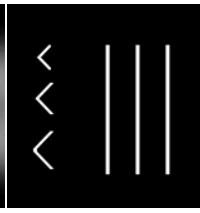

(c)

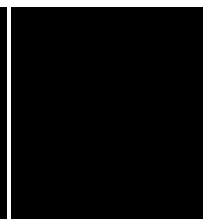

(g)

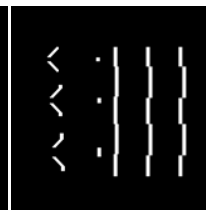

(d)

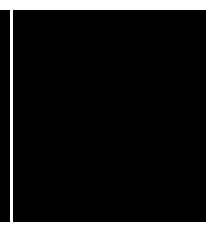

(h)
Figure 3. The environmental adaptive threshold and the response of left tremor of a horizontal ATO-DPAM-m detector with different phases and amplitudes for video flow $u_{p}(k)=I_{p+k v}^{\prime}$ formed by a left moving image $I^{\prime}$ with velocity $v$. (a) a stripe image example in the video flow; (b) the environmental adaptive threshold; (c) and (d) demonstrate the ATO-R response yielded by $\tilde{G}_{\text {. }}^{+}(k)$ output for in-phase edges and that yielded by $\tilde{G}_{-}^{-}(k)$ output for antiphase edges respectively when the ATO phase is $\pi / 2$; (e) and (f) show the ATO-R response yielded by $\tilde{G}_{.}^{+}(k)$ output for in-phase edges and that yielded by $\tilde{G}_{-}^{-}(k)$ output for antiphase edges respectively when the ATO amplitude $\Delta d>v$ and the phase is $3 \pi / 2$. (g) and (h) show the visual fading on the output of $\tilde{G}_{.}^{+}(k)$ and $\tilde{G}_{.}^{-}(k)$ respectively when the ATO amplitude $\Delta d=v$ and the ATO phase is $3 \pi / 2$.
According to the property of in-phase enhancement and anti-phase inhibition, we compare the different combinations of output response $\tilde{\mathbf{G}}_{\bullet}^{+}(k)$ and $\tilde{\mathbf{G}}_{\bullet}^{-}(k)$, yielding a new set of outputs, i.e.

$$
\left\{\begin{array}{l}
\mathbf{G}_{\mathrm{P}}^{+}(k)=\tilde{\mathbf{G}}_{\mathrm{P}}^{+}(k)-\tilde{\mathbf{G}}_{\mathrm{N}}^{+}(k)-\tilde{\mathbf{G}}_{\mathrm{N}}^{-}(k) \\
\mathbf{G}_{\mathrm{P}}^{-}(k)=\tilde{\mathbf{G}}_{\mathrm{P}}^{-}(k)-\tilde{\mathbf{G}}_{\mathrm{N}}^{+}(k)-\tilde{\mathbf{G}}_{\mathrm{N}}^{-}(k) \\
\mathbf{G}_{\mathrm{N}}^{+}(k)=\tilde{\mathbf{G}}_{\mathrm{N}}^{+}(k)-\tilde{\mathbf{G}}_{\mathrm{P}}^{+}(k)-\tilde{\mathbf{G}}_{\mathrm{P}}^{-}(k) \\
\mathbf{G}_{\mathrm{N}}^{-}(k)=\tilde{\mathbf{G}}_{\mathrm{N}}^{-}(k)-\tilde{\mathbf{G}}_{\mathrm{P}}^{+}(k)-\tilde{\mathbf{G}}_{\mathrm{P}}^{-}(k)
\end{array}\right.
$$

where $\mathbf{G}_{\mathrm{P}}^{+}(k)$ and $\mathbf{G}_{\mathrm{P}}^{-}(k)$ denote the positive On-type output and Off-type output respectively. They selectively respond to On-type and Off-type moving edges whose moving directions are not identical to the positive direction of the ATO. $\mathbf{G}_{\mathrm{N}}^{+}(k)$ and $\mathbf{G}_{\mathrm{N}}^{-}(k)$ represent the negative On-type and Off-type output respectively. They selectively respond to On-type and Off-type moving edges whose moving directions are not identical to the negative direction of the ATO. As shown in Figure 5, the different outputs of the proposed AMD can selectively respond to the edges with specific motion characteristics.

In summary, the proposed AMD has realized a combinational selective detector to capture moving edges based on the ATO resonance enhancement and inhibition of edges in different moving directions. The method is robust to backgrounds with change or switch due to the environmental adaptive threshold.

\section{Experimental Results and Analysis}

In this section, we experiment using the KTH dataset [17] and analyze the performance of the proposed adaptive

$u(k)$

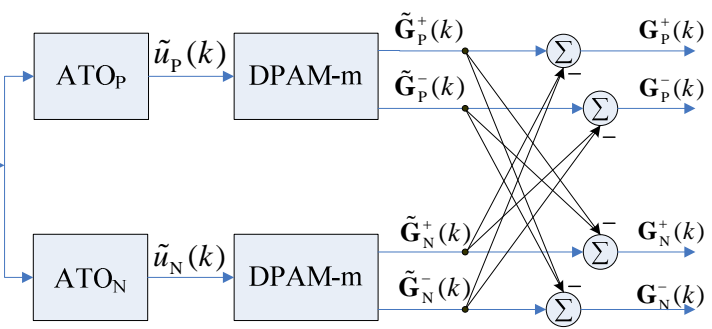

Figure 4. The diagram of ATO-AMD's configuration and its output connection.

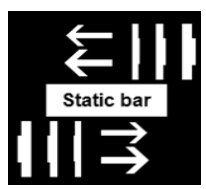

(a)

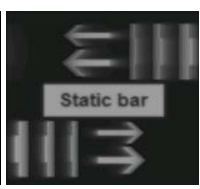

(b)

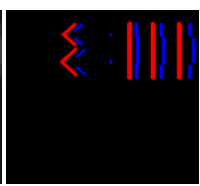

(c)

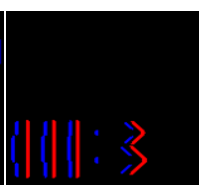

(d)
Figure 5. The AMD response to a synthesized video input. (a) is the synthesized video input; (b) shows the adaptive threshold $A(k)$; (c) and (d) shows respectively the positive and negative direction responses, where the red regions stand for On-type and the blue regions for Off-type. 
motion detector (AMD). A number of behavior analysis results have been reported based on the KTH dataset. However their analysis involves only motion perception and motion feature extraction.

We construct a four-directional motion detector combination which is formed by a pair of horizontal AMDs and a pair of vertical AMDs to detect motion in $\mathrm{KTH}$ videos with the movements of jogging, arm lift, and arm down. See in Figures 6, 7 and $\mathbf{8}$ for detection results: (a) a frame of input video; (b) the environmental adaptive threshold A(k); (c) and (f) show the vertical edge responses, upward and downward respectively; (d) and (e) illustrate the horizontal edge responses, towards the left and the right respectively. What's more, the red regions denote On-type moving edges and the blue regions denote Off-type moving edges.

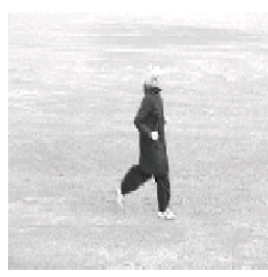

(a)

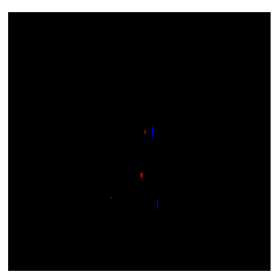

(d)

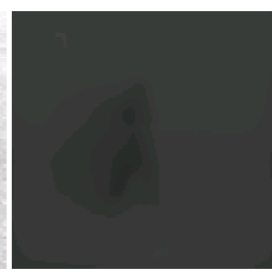

(b)

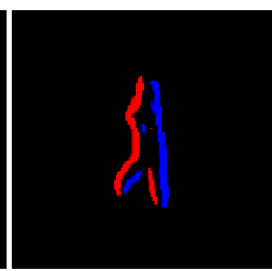

(e)

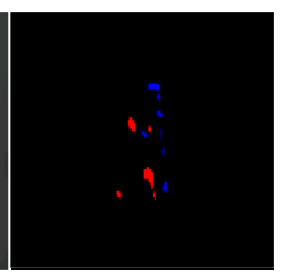

(c)

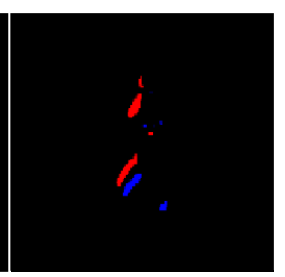

(f)
Figure 6. Detecting results of ATO-AMD on a video with a body moving to the right.

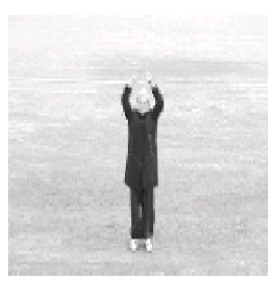

(a)

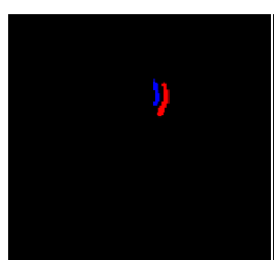

(d)

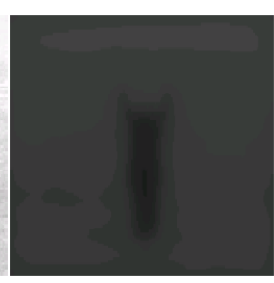

(b)

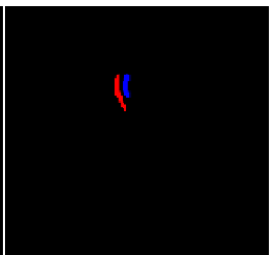

(e)

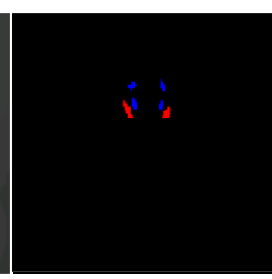

(c)

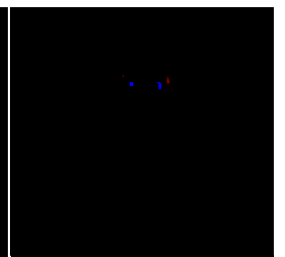

(f)
Figure 7. Detecting results of ATO-AMD on a video with arm lift movement.

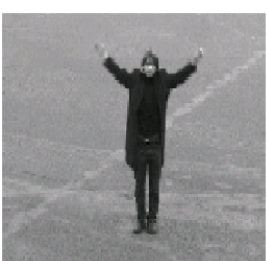

(a)

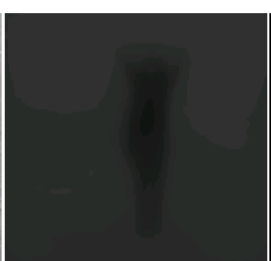

(b)

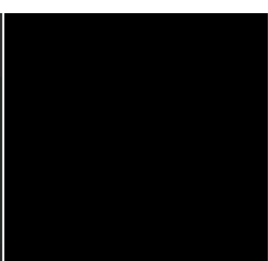

(c)

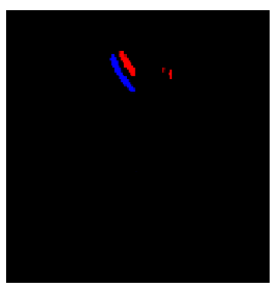

(d)

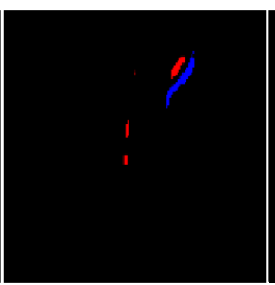

(e)

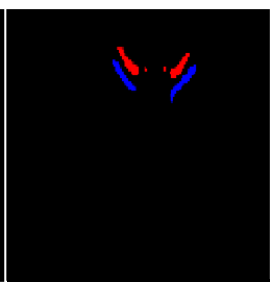

(f)
Figure 8. Detecting results of ATO-AMD on a video with arm dropped down.

From the results, we find this four-directional detector combination can detect effectively both On-type and Offtype motion with different moving directions. For the movements of jogging towards the right, the detector barely responds to the movements towards the left, e.g. Figure 6(d), while the detector for movements towards the right brings obvious output response, e.g. Figure 6(e). On the other hand, there occurs a little upward and downward movement simultaneously with left-right movement. Therefore the detectors for upward and downward motion can respond a bit to left-right movement. Likewise, the other detectors generate similar results shown in Figures 6,7 and 8 .

\section{Conclusions}

Inspired by the neuroscience research, in this paper we proposed a so-called dual-probe adaptive model (DPAM) and an active tremor operation (ATO) approach to simulate the visual attention selection process and the fixational eye movement. We found that they enhance each other if in-phase and inhibit each other if anti-phase. Based on this finding, we has constructed a framework by combining DPAMs and ATOs in two opposite directions. This framework can agilely capture the edges with different moving directions even in the image sequences with lighting change and noisy background. Therefore, the proposed method provide an important basis for the further study on motion analysis system.

\section{Acknowledgements}

This work was supported by the grants from Natural Science Foundation of Shenzhen University (grant no. 201206). The authors would also like to thank the anonymous reviewers. 


\section{REFERENCES}

[1] C. W. G. Clifford and M. R. Ibbotson, "Fundamental mechanisms of visual motion detection: models, cells and functions,” Progress in Neurobiology, Vol. 68, No. 6, 2002, pp. 409-437. doi:10.1016/S0301-0082(02)00154-5

[2] A. J. Lipton, H. Fujiyoshi and R. S. Patil, "Moving Target Classification and Tracking from Real-time Video," in IEEE Workshop on Applications of Computer Vision, 1998, pp. 8-14.

[3] Y. Ren, C.-S. Chua and Y.-K. Ho, "Motion detection with nonstationary background," Machine Vision and Applications, Vol. 13, No. 5, 2003, pp. 332-343. doi:10.1007/s00138-002-0091-0

[4] D. Marr and S. Ullman, "Directional Selectivity and Its Use in Early Visual Processing," Proceedings of the Royal Society of London. Series B, Vol. 211, 1981, pp. 151-180. doi:10.1098/rspb.1981.0001

[5] B. K. P. Horn and B. G. Schunck, "Determining Optical Flow,” Artificial Intelligence, Vol. 17, 1981, pp. 185-203. doi:10.1016/0004-3702(81)90024-2

[6] A. Bouzerdoum and R. B. Pinter, “A Shunting Inhibitory Motion Detector that Can Account for the Functional Characteristics of Fly Motion-Sensitive Interneurons,” in 1990 IJCNN International Joint Conference on Neural Networks, Vol.1, 1990, pp. 149-153. doi:10.1109/IJCNN.1990.137560

[7] A. Bouzerdoum, "The Elementary Movement Detection Mechanism in Insect Vision,” Philosophical Transactions: Biological Sciences, Vol. 339, No. 1290, 1993, pp. 375-384.doi:10.1098/rstb.1993.0037

[8] A. Wang, N. Zheng, L. Yuan et al., "Multiplicative Inhibitory Velocity Detector (MIVD) and Multi-Velocity Motion Detection Neural Network Model,” Proceedings of International Conference on Multi-Sensor Fusion and Integration for Intelligent Systems, 1996, pp. 476-483.

[9] P. Sterling, "Microcircuitry of the Cat Retina," Annual Review of Neuroscience, Vol. 6, No. 1, 1983, pp. $149 \mathrm{C} 185$.
[10] L. Ma, N.-N. Zheng and Z.-J. Yuan, “A Dynamic Model for Early Vision Processing,” in IEEE International Conference on Computer and Electrical Engineering, Phuket Island, Thailand, 2008, pp. 96-100.

[11] L. Ma, N.-N. Zheng, Z.-J. Yuan et al., "A Novel Dual Probes Adaptive Model for Image Change Detection,” IEEE Signal Proceeding Letters, Vol. 17, No. 10, 2010, pp. 863-866. doi:10.1109/LSP.2010.2063023

[12] S. Martinez-Conde, S. L. Macknik, and D. H. Hubel, "The Role of Fixational Eye Movements in Visual Perception,” Nature Reviews Neuroscience, Vol. 5, No. 3, 2004, pp. 229-240. doi:10.1038/nrn1348

[13] I. Murakami, "Fixational Eye Movements and Motion Perception," in Twenty-eighth European Conference on Visual Perception, A Coruna, Spain, 2005, pp. 35-35.

[14] S. Moshel, A. Z. Zivotofsky, L. Jin-Rong et al., "Persistence and Phase Synchronisation Properties of Fixational Eye Movements,” European Physical Journal-Special Topics, Vol. 161, Jul, 2008, pp. 207-223. doi:10.1140/epjst/e2008-00762-3

[15] M. Greschner, M. Bongard, P. Rujan et al., "Retinal Ganglion Cell Synchronization by Fixational Eye Movements Improves Feature Estimation,” Nature Neuroscience, Vol. 5, No. 4, 2002, pp. 341-347. doi:10.1038/nn821

[16] Engbert, Ralf. “Computational Modeling of Collicular Integration of Perceptual Responses and Attention in Microsaccades,” The Journal of Neuroscience, Vol. 32, No. 23, 2012, pp. 8035-8039. doi:10.1523/JNEUROSCI.0808-12.2012

[17] Z. Gao, M. Chen, et al. "Comparing Evaluation Protocols on the KTH Dataset," Human Behavior Understanding (2010): 88-100.

[18] W. Hui, Q. Zuo and X. Guan, "Main Retina Information Processing Pathways Modeling," International Journal of Cognitive Informatics and Natural Intelligence, Vol. 5, No. 3, 2011, pp. 30-46. doi:10.4018/IJCINI.2011070102 